\title{
Observation of the black widow B1957+20 millisecond pulsar binary system with the MAGIC telescopes
}

M. L. Ahnen, ${ }^{1}$ S. Ansoldi, ${ }^{2,3}$ L. A. Antonelli, ${ }^{4}$ C. Arcaro, ${ }^{5}$ A. Babić, ${ }^{6}$ B. Banerjee, ${ }^{7}$ P. Bangale, ${ }^{8}$ U. Barres de Almeida, ${ }^{8,9}$ J. A. Barrio, ${ }^{10}$ J. Becerra González, ${ }^{11,12,13,14}$ W. Bednarek, ${ }^{15 \star}$ E. Bernardini, ${ }^{16,17}$ A. Berti, ${ }^{2,18}$ B. Biasuzzi, ${ }^{2}$ A. Biland, ${ }^{1}$ O. Blanch, ${ }^{19}$ S. Bonnefoy, ${ }^{10}$ G. Bonnoli, ${ }^{20}$ F. Borracci, ${ }^{8}$ T. Bretz,${ }^{21}$ R. Carosi,${ }^{20}$ A. Carosi,${ }^{4}$ A. Chatterjee, ${ }^{7}$ P. Colin,${ }^{8}$ E. Colombo, ${ }^{11,12}$ J. L. Contreras, ${ }^{10}$ J. Cortina, ${ }^{19}$ S. Covino,${ }^{4}$ P. Cumani, ${ }^{19}$ P. Da Vela, ${ }^{20}$ F. Dazzi, ${ }^{4}$ A. De Angelis, ${ }^{5}$ B. De Lotto, ${ }^{2}$ E. de Oña Wilhelmi, ${ }^{22}$ F. Di Pierro, ${ }^{4}$ M. Doert, ${ }^{23}$ A. Domínguez, ${ }^{10}$ D. Dominis Prester, ${ }^{6}$ D. Dorner, ${ }^{21}$ M. Doro, ${ }^{5}$ S. Einecke, ${ }^{23}$ D. Eisenacher Glawion, ${ }^{21}$ D. Elsaesser, ${ }^{23}$ M. Engelkemeier, ${ }^{23}$ V. Fallah Ramazani, ${ }^{24}$ A. Fernández-Barral, ${ }^{19}$ D. Fidalgo, ${ }^{10}$ M. V. Fonseca, ${ }^{10}$ L. Font,${ }^{26}$ C. Fruck, ${ }^{8}$ D. Galindo, ${ }^{26}$ R. J. García López, ${ }^{11,12}$ M. Garczarczyk, ${ }^{16}$ M. Gaug, ${ }^{25}$ P. Giammaria, ${ }^{4}$ N. Godinović, ${ }^{6}$ D. Gora, ${ }^{16}$ S. R. Gozzini, ${ }^{16}$ S. Griffiths, ${ }^{19}$ D. Guberman, ${ }^{19}$ D. Hadasch, ${ }^{27}$ A. Hahn, ${ }^{8}$ T. Hassan, ${ }^{19}$ M. Hayashida, ${ }^{27}$ J. Herrera, ${ }^{11,12}$ J. Hose, ${ }^{8}$ D. Hrupec, ${ }^{6}$ G. Hughes, ${ }^{1}$ K. Ishio,${ }^{8}$ Y. Konno, ${ }^{27}$ H. Kubo, ${ }^{27}$ J. Kushida,${ }^{27}$ D. Kuveždić, ${ }^{6}$ D. Lelas, ${ }^{6}$ E. Lindfors, ${ }^{24}$ S. Lombardi, ${ }^{4}$ F. Longo, ${ }^{2,18}$ M. López, ${ }^{10 \star}$ P. Majumdar, ${ }^{7}$ M. Makariev,${ }^{28}$ G. Maneva, ${ }^{28}$ M. Manganaro, ${ }^{11,12}$ K. Mannheim, ${ }^{21}$ L. Maraschi, ${ }^{4}$ M. Mariotti, ${ }^{5}$ M. Martínez, ${ }^{19}$ D. Mazin, ${ }^{3,8}$ U. Menzel, ${ }^{8}$ R. Mirzoyan, ${ }^{8}$ A. Moralejo, ${ }^{19}$ V. Moreno, ${ }^{25}$ E. Moretti, ${ }^{8}$ V. Neustroev, ${ }^{24}$ A. Niedzwiecki, ${ }^{15}$ M. Nievas Rosillo, ${ }^{10}$ K. Nilsson, ${ }^{24}$ K. Nishijima, ${ }^{27}$ K. Noda, ${ }^{8}$ L. Nogués, ${ }^{19}$ S. Paiano,${ }^{5}$ J. Palacio, ${ }^{19}$ D. Paneque, ${ }^{8}$ R. Paoletti, ${ }^{20}$ J. M. Paredes, ${ }^{26}$ X. Paredes-Fortuny,${ }^{26}$ G. Pedaletti, ${ }^{16}$ M. Peresano, ${ }^{2}$ L. Perri, ${ }^{4}$ M. Persic, ${ }^{2}$ J. Poutanen, ${ }^{24}$ P. G. Prada Moroni, ${ }^{29}$ E. Prandini,${ }^{5}$ I. Puljak, ${ }^{6}$ J. R. Garcia, ${ }^{8}$ I. Reichardt, ${ }^{5}$ W. Rhode, ${ }^{23}$ M. Ribó,${ }^{26}$ J. Rico, ${ }^{19}$ T. Saito, ${ }^{27}$ K. Satalecka, ${ }^{16}$ S. Schroeder, ${ }^{23}$ T. Schweizer, ${ }^{8}$ A. Sillanpää, ${ }^{24}$ J. Sitarek, ${ }^{15 \star}$ I. Šnidarić, ${ }^{6}$ D. Sobczynska, ${ }^{15}$ A. Stamerra, ${ }^{4}$ M. Strzys,${ }^{8}$ T. Surić,${ }^{6}$ L. Takalo,${ }^{24}$ F. Tavecchio, ${ }^{4}$ P. Temnikov, ${ }^{28}$ T. Terzić, ${ }^{6}$ D. Tescaro,${ }^{5}$ M. Teshima, ${ }^{3,8}$ D. F. Torres,${ }^{30}$ N. Torres-Albà ${ }^{26}$ A. Treves, ${ }^{2}$ G. Vanzo, ${ }^{11,12}$ M. Vazquez Acosta, ${ }^{11,12}$ I. Vovk, ${ }^{8}$ J. E. Ward ${ }^{19}$ M. Will, ${ }^{11,12}$ M. H. Wu, ${ }^{22}$ D. Zarić, ${ }^{6}$ (MAGIC Collaboration), ${ }^{6}$ I. Cognard ${ }^{31,32}$ and L. Guillemot ${ }^{31,32}$

Affiliations are listed at the end of the paper 


\begin{abstract}
B1957+20 is a millisecond pulsar located in a black-widow-type compact binary system with a low-mass stellar companion. The interaction of the pulsar wind with the companion star wind and/or the interstellar plasma is expected to create plausible conditions for acceleration of electrons to $\mathrm{TeV}$ energies and subsequent production of very high-energy $\gamma$-rays in the inverse Compton process. We performed extensive observations with the Major Atmospheric Gamma Imaging Cherenkov Telescopes (MAGIC) telescopes of B1957+20. We interpret results in the framework of a few different models, namely emission from the vicinity of the millisecond pulsar, the interaction of the pulsar and stellar companion wind region or bow shock nebula. No significant steady very high-energy $\gamma$-ray emission was found. We derived a 95 per cent confidence level upper limit of $3.0 \times 10^{-12} \mathrm{~cm}^{-2} \mathrm{~s}^{-1}$ on the average $\gamma$-ray emission from the binary system above $200 \mathrm{GeV}$. The upper limits obtained with the MAGIC constrain, for the first time, different models of the high-energy emission in B1957+20. In particular, in the inner mixed wind nebula model with mono-energetic injection of electrons, the acceleration efficiency of electrons is constrained to be below $\sim 2-10$ per cent of the pulsar spin-down power. For the pulsar emission, the obtained upper limits for each emission peak are well above the exponential cut-off fits to the Fermi-LAT data, extrapolated to energies above $50 \mathrm{GeV}$. The MAGIC upper limits can rule out a simple power-law tail extension through the sub-TeV energy range for the main peak seen at radio frequencies.
\end{abstract}

Key words: radiation mechanisms: non-thermal - binaries: close-pulsars: general-pulsars: individual: B1957+20 - gamma-rays: general.

\section{INTRODUCTION}

$\mathrm{B} 1957+20$ is a millisecond pulsar (MSP), with a period of $1.6 \mathrm{~ms}$ and a surface magnetic field of $\sim 10^{8} \mathrm{G}$. It is located within a very compact binary system of the black-widow-type with a period of $9.2 \mathrm{~h}$ (Fruchter et al. 1988). The black widow binary systems contain a very low mass companion star that is being evaporated due to the energy release by the MSP. In the case of B1957+20, the companion star has the mass of $\sim 0.022 \mathrm{M}_{\odot}$ (van Paradijs et al. 1988) and a radius of $\sim 10^{10} \mathrm{~cm}$. Its surface temperature varies between 2900 and $8300 \mathrm{~K}$ for the side illuminated by the pulsar (Fruchter et al. 1996; Reynolds et al. 2007). The pulsed radio emission of $\mathrm{B} 1957+20$ is eclipsed for about 10 percent of the orbital phase by the wind from the companion star. The wind region is limited by the shock formed in the interaction of the MSP and companion winds within the binary system (Fruchter et al. 1988). The existence of the wind collision shock is also supported by the observations of the X-ray emission, modulated with the period of the binary system (Huang et al. 2012). The model for the X-ray emission in the wind shock scenario has been considered by Harding \& Gaisser (1990) and Arons \& Tavani (1993) and more recently by Wadiasingh et al. (2015).

The binary system $\mathrm{B} 1957+20$ is surrounded by an $\mathrm{H} \alpha$ emission nebula that is expected to be produced in the interaction of the pulsar wind with the interstellar medium (Kulkarni \& Hester 1988). The nebula reveals a bow shock around the system, which moves through the medium with a velocity of $\sim 220 \mathrm{~km} \mathrm{~s}^{-1}$, based on the proper motion measurements (Arzoumanian et al. 1994). The extended $\mathrm{X}$-ray emission has also been reported from the interior of the bow shock (Stappers et al. 2003; Huang \& Becker 2007; Huang et al. 2012). This non-thermal X-ray emission creates a tail behind the moving pulsar with a length of $\sim 10^{18} \mathrm{~cm}$. The extended emission is interpreted as produced by relativistic electrons accelerated in the pulsar vicinity (Cheng, Taam \& Wang 2006).

This binary system was claimed in the past to be a GeV-TeV $\gamma$-ray source by the Potchefstroom air Cerenkov telescope and the
COS B satellite (Brink et al. 1990). These findings have not been confirmed by the Potchefstroom/Nooitgedacht (Raubenheimer et al. 1995) and the EGRET observations (Buccheri et al. 1996). Such potential, non-pulsed emission might be produced by the Comptonization of the companion star radiation by leptons accelerated in the collision region between the pulsar wind and the donor star wind (Raubenheimer et al. 1995). A pulsed GeV $\gamma$-ray emission was discovered by the Fermi-LAT (Guillemot et al. 2012). This emission shows two characteristic peaks in the light curve. The spectrum is well described by a power law with an exponential cut-off at the energy of a few GeV. Similar cut-offs have also been observed in classical pulsars. Therefore, the particle acceleration processes within inner pulsar magnetospheres can be similar for classical and MSPs. These similarities may also indicate that the properties of the winds produced by the millisecond and classical pulsars are likely similar. In fact, the classical pulsar PSR B1259-63, found within the binary system on the orbit around a Be-type star, has been reported to emit $\mathrm{TeV} \gamma$-rays close to the periastron passage (Aharonian et al. 2005). The discovery of $\mathrm{TeV}$ emission from a binary system containing MSPs will put a new constraint on the acceleration and radiation processes of energetic particles in the vicinity of pulsars. Due to the different compactness of the $\mathrm{TeV} \gamma$-ray and MSP type of binaries, as well as the different radiation fields produced by the companion stars, the detection of $\mathrm{TeV} \gamma$-rays could provide new insight into the structure of the pulsar winds, their magnetization and localization of acceleration regions outside the light cylinder radius.

The existence of an additional higher energy $\mathrm{GeV} \gamma$-ray component, which appears at a specific range of the orbital phases, has been also reported based on the analysis of the Fermi-LAT data (Wu et al. 2012). This component can be modelled by electrons from the pulsar Comptonizing the radiation from the companion star.

The variety of the non-thermal phenomena observed in the MSP binary system B1957+20 suggests that the conditions in its vicinity are suitable for production of very high-energy $(E>100 \mathrm{GeV})$ $\gamma$-rays. In this paper, we present the results of the very high-energy 


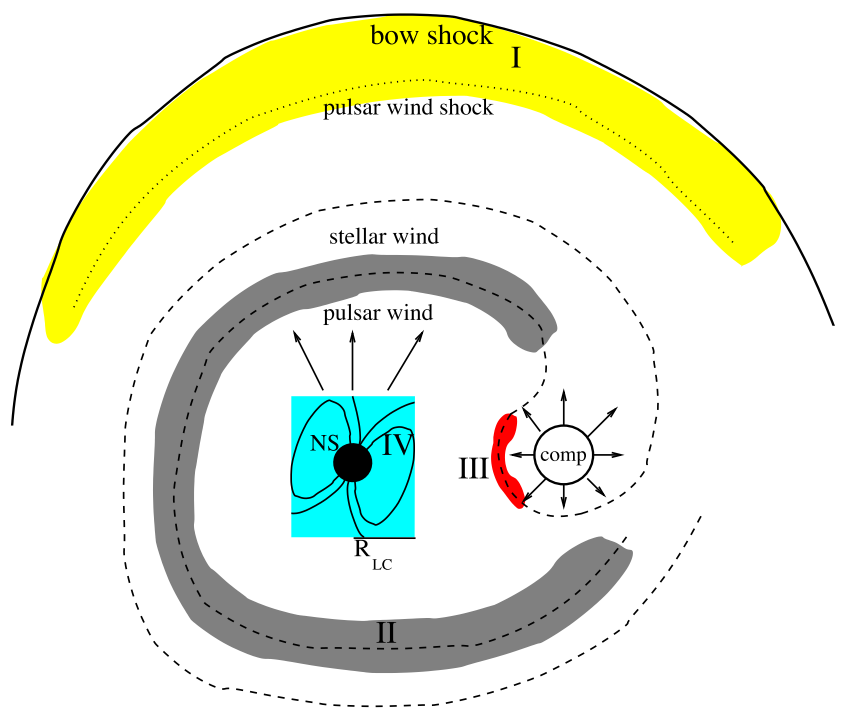

Figure 1. Schematic cartoon showing zones of various scenarios of $\gamma$-ray emission from the B1957+20 system. The emission associated with the pulsar wind shock within the bow shock of the binary occurs in region I (yellow, see Section 5.1). In the region II (in grey), mixing of the pulsar wind with the wind of its stellar companion occurs outside of the binary system (see Section 5.2). In the region III, a modulated emission can be produced from the direct interaction, within the binary system, of the two winds (red, see Section 5.3). The emission might be also generated in the vicinity of the pulsar (cyan, IV, see Section 5.4).

range $\gamma$-ray observations of B1957+20 performed with the Major Atmospheric Gamma Imaging Cherenkov Telescopes (MAGIC) telescopes. The derived upper limits on the high-energy $\gamma$-ray emission from the nebula around the binary system, from the binary system itself and from the MSP B1957+20, are discussed in the context of available theoretical models. In particular, we consider a possible production of the high-energy $\gamma$-rays by electrons accelerated on the large-scale MSP shock in the pulsar wind within the bow shock nebula; on the shocks resulted from the mixture of the pulsar and companion star winds in the equatorial region of the binary system (they are twisted around the binary system due to the Coriolis force); on the shock in the inner part of the binary system formed between the companion star and the pulsar, and also $\gamma$-rays that might be produced in the region close to the MSP light cylinder or in the MSP inner magnetosphere. The different emission zones occurring in those models are schematically depicted in Fig. 1.

\section{MAGIC DATA AND ANALYSIS}

MAGIC is a system of two Imaging Atmospheric Cherenkov Telescopes located at Roque de los Muchachos, on the Canary island of La Palma, Spain (Aleksić et al. 2016a). It is used for observations of $\gamma$-rays with energies above $\sim 50 \mathrm{GeV}$. It has a sensitivity of $(0.66$ \pm 0.03 ) per cent of C.U. (Crab nebula flux) in $50 \mathrm{~h}$ of observations for energies above $220 \mathrm{GeV}$ (Aleksić et al. 2016b). The angular resolution (defined as a standard deviation of a two-dimensional Gaussian distribution) at those energies is $\lesssim 0.07$.

B1957+20 was observed by the MAGIC telescopes between 2013 June and October. The data were taken at low and medium zenith angles, $8^{\circ}-52^{\circ}$, with most of the data taken below $30^{\circ}$. We analysed the data using the standard MAGIC analysis chain (Zanin et al. 2013; Aleksić et al. 2016b). To the data affected by non-perfect atmospheric conditions, we have applied a correction based on simultaneous Light Detection and Ranging measurements (Fruck \& Gaug 2015).

Together with each event image, we recorded the absolute event arrival time using a GPS receiver. The absolute time precision of the events registered by MAGIC is $200 \mathrm{~ns}$.

For the analysis of the binary system, we have selected $66.5 \mathrm{~h}$ of data based on the rate of background events and atmospheric transmission from a height of $9 \mathrm{~km}$ being above 0.6 with respect to the perfect atmospheric conditions. We performed analysis in bins of orbital period using ephemeris of Guillemot et al. (2012). The orbital phase is defined starting from the ascending node with respect to the MSP, i.e. phase 0.75 corresponds to the pulsar between the companion and the observer. For the pulsar analysis, however, tighter cuts were applied to ensure the lowest possible energy threshold. Data taken at zenith angles above $30^{\circ}$ were discarded as well as those with too low atmospheric transmission for pulsar studies (the integrated transmission above $9 \mathrm{~km}$ exceeding 0.8 of the one with perfect atmospheric conditions). The observation time for the pulsar analysis is $29.4 \mathrm{~h}$.

The pulsar rotational phase of the events was computed using Tempo2 (Hobbs, Edwards \& Manchester 2006), and an ephemeris for PSR B1957+20 covering the MAGIC observations. The ephemeris was constructed by analysing pulsar timing data recorded at the Nançay radio telescope, near Orléans, France. The radio data were scrunched in frequency and in polarization, and were integrated in time in order to build one pulsar profile every $10 \mathrm{~min}$ of observation. A total of 234 times of arrival were then extracted from these data and analysed with Tempo2 to obtain a timing solution for PSR B 1957+20 covering the MJD period 56 048-57 270. The timing solution uses the BTX binary model and three orbital frequency derivatives, to track changes in the orbital period of the system accurately. The weighted $\mathrm{rms}$ of the timing residuals is $2.5 \mu \mathrm{s}$. Note that this ephemeris has a different phase 0 definition than that used in Guillemot et al. (2012).

We compute the significance using equation 17 in $\mathrm{Li} \& \mathrm{Ma}$ (1983). All upper limits on the flux presented in this paper were calculated following the approach of Rolke, López \& Conrad (2005) using 95 per cent confidence level and assuming 30 per cent total systematic uncertainty. For calculation of upper limits on the flux from the binary system, in addition we require that the upper limit on the number of excess events is above 3 per cent of the residual background. This ensures that the background induced systematic uncertainties do not exceed the claimed above total systematic uncertainty (see Aleksić et al. 2016b). Such additional condition is not needed in the case of pulsar analysis, as the off-pulse background estimation is expected to have a negligible effect on total systematic uncertainty.

\section{FERMI-LAT DATA AND ANALYSIS}

A data sample of more than $3 \mathrm{yr}$ (from 56048 up to 57270 MJD) of Fermi-LAT data was analysed, according to the period of validity of the ephemeris used. We analysed this data set using the P8R2_SOURCE_V6 instrument response functions and the Fermi Science Tools version v10r0p $5^{1}$. Events were selected within a circular region of interest (ROI) of $15^{\circ}$ centred at the pulsar position (R.A. $=19^{\mathrm{h}} 59^{\mathrm{m}} 36.77$, Dec $=20^{\circ} 48^{\prime} 15^{\prime \prime}$. 12). We selected 'Source' class events (evclass $=128$ and evtype $=3$ ) that were recorded only

\footnotetext{
${ }^{1}$ Available online at http://fermi.gsfc.nasa.gov/ssc/data/analysis/software/.
} 
Table 1. Definition of the signal and background regions derived from the Fermi -LAT data.

\begin{tabular}{ccc}
\hline P1 & P2 & Off-region \\
\hline$-0.016-0.008$ & $0.520-0.563$ & $0.64-0.94$ \\
\hline
\end{tabular}

when the telescope was in nominal science mode. To reject the background coming from the Earth's limb, we selected photons with a zenith angle $\leq 90^{\circ}$. The pulsar rotational phase and barycentric corrections of the events were computed using Tempo 2 with the Fermi plug-in ${ }^{2}$, using the same ephemeris as for MAGIC data analysis. The pulsar light curve was produced by applying an additional energy dependent angular cut defined by: $R=\max \left(0.8(E / 1 \mathrm{GeV})^{-0.8}\right.$, $0.2)^{\circ}$, according to the approximation of the Fermi-LAT Pass8 point spread function for a 68 per cent confinement radius (Acero et al. 2015).

For the spectral analysis, a binned likelihood analysis was performed making use of the pyLikelihood python module of the Fermi tools, for both peaks, P1 and P2, separately. We started by including all sources in ROI from the third Fermi Source Catalog (Acero et al. 2015) in the spectral-spatial model, resulting in a total of 174 sources. All sources from 3FGL catalogue were assumed to have the spectral type as suggested in the catalogue. The spectral parameters for sources with a significance higher than $5 \sigma$ and located at less than $5^{\circ}$ away from the ROI centre were left free. We also let the normalization factor of the isotropic (iso_P8R2_SOURCE_V6_v06.txt) and Galactic (gll_iem_v06.fits) background models free. For the rest of the sources, all parameters were left fixed to their catalogue value. Finally, all sources with TS $<4$ were removed from the model, reducing the number of sources to 66 and the number of free parameters to 13. For the calculation of the spectral points, we repeated the procedure in each energy bin using a power law with the normalization factor free and a spectral index fixed to 2 . Whenever the significance of the spectral point was less than $1.5 \sigma$, a 95 per cent confidence level upper limit was calculated instead.

In order to search for a possible nebula emission at $\mathrm{GeV}$ energies, we performed a spectral fit to the photons falling in the off-pulse interval (defined in Table 1), where no pulsar emission is expected. For this fit, we used a model in which the pulsar component is removed and the nebula is described by a power-law point-like source.

\section{RESULTS}

We have performed an analysis of the MAGIC data searching for a steady emission from the black widow system. The pulsed emission is investigated using MAGIC and Fermi-LAT data.

\subsection{Analysis of the nebular emission}

Using the standard detection cuts of $\mathrm{MAGIC}^{3}$, no significant $\gamma$ ray emission has been found from the direction of B1957+20 in the analysis of the MAGIC data (see Fig. 2). For the zenith angle distribution of the B1957+20 observations, these cuts correspond to an energy threshold of $\sim 250 \mathrm{GeV}$. There is also no significant emission in the region around the nominal position of the source, as seen in the skymap of Fig. 3.

\footnotetext{
${ }^{2}$ http://fermi.gsfc.nasa.gov/ssc/data/analysis/user/Fermi_plug_doc.pdf.

${ }^{3}$ They have been selected such as to provide optimal sensitivity over a broad range of possible spectral shapes of the source.
}

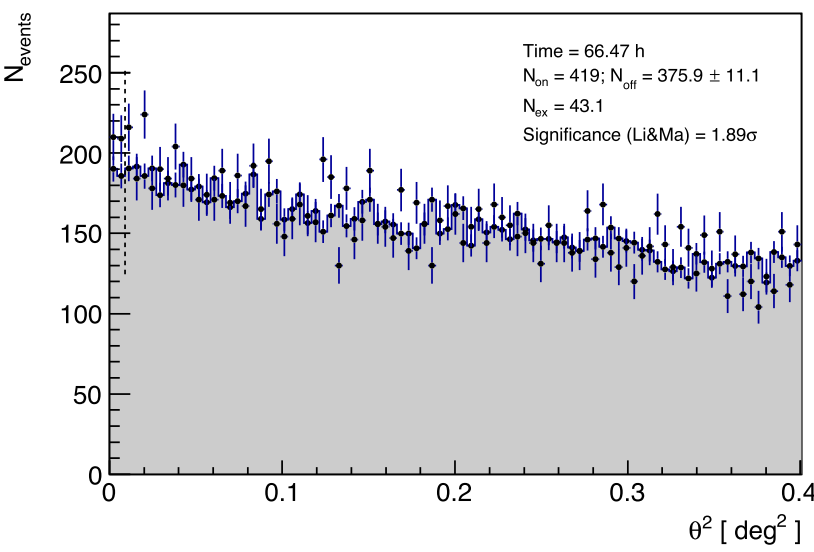

Figure 2. Distribution of the squared angular distance between the reconstructed event direction and the nominal source position (points) and the background estimation (shaded area). The corresponding energy threshold (defined as the peak of the differential energy distribution for Monte Carlo $\gamma$-rays with a spectral slope of -2.6 ) is $\sim 250 \mathrm{GeV}$.

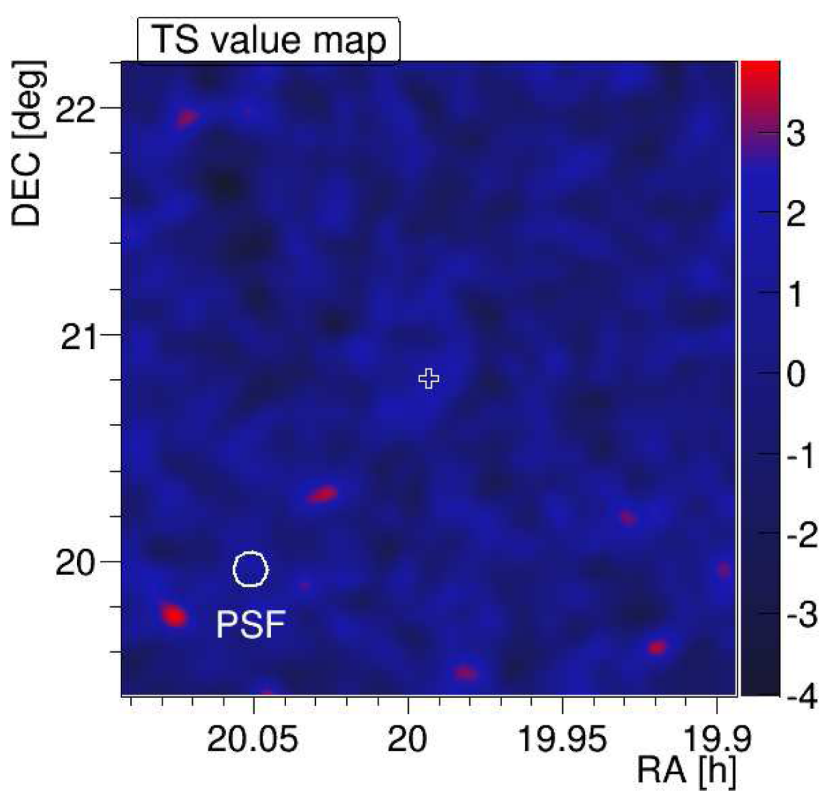

Figure 3. Significance skymap of the region around the position of B1957+20 (marked with an empty cross). The corresponding energy threshold is $\sim 250 \mathrm{GeV}$.

Let us first assume that we can treat the source as point-like (i.e. its radius should be much smaller than the angular resolution of MAGIC at those energies, 0.07 ). Using the whole data set, we obtain an upper limit on the flux above $200 \mathrm{GeV}$ of $3.0 \times$ $10^{-12} \mathrm{~cm}^{-2} \mathrm{~s}^{-1}$ (i.e. $\sim 1.3$ percent C.U.). This value is rather high compared with the sensitivity of MAGIC telescopes above those energies due to a small positive excess that increases the value of the upper limit. Using the complete data set, we also computed upper limits on the flux in differential energy bins (five per decade). We present them in the spectral energy distribution (SED) form in Fig. 4.

We also performed an analysis dividing the total data set into six bins according to the orbital phase of the binary system. As can be seen in the top panel of Fig. 5, the MAGIC observations covered the whole orbit of the system homogeneously. The highest obtained significance, $2.5 \sigma$, occurred in the orbital phase bin of 


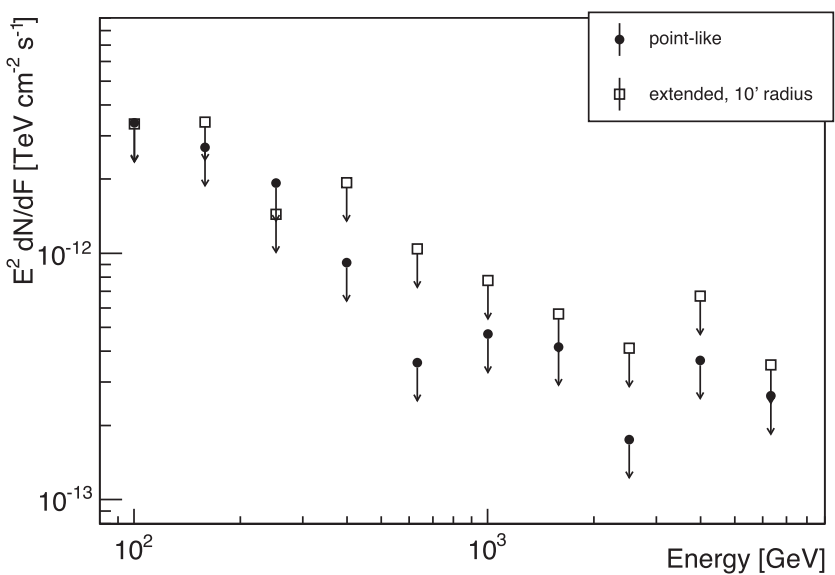

Figure 4. 95 per cent confidence level upper limits on the level of spectral energy distribution of B1957+20 computed under the assumption of a pointlike source (filled circles) and an extended source with 10 arcmin radius (open squares).

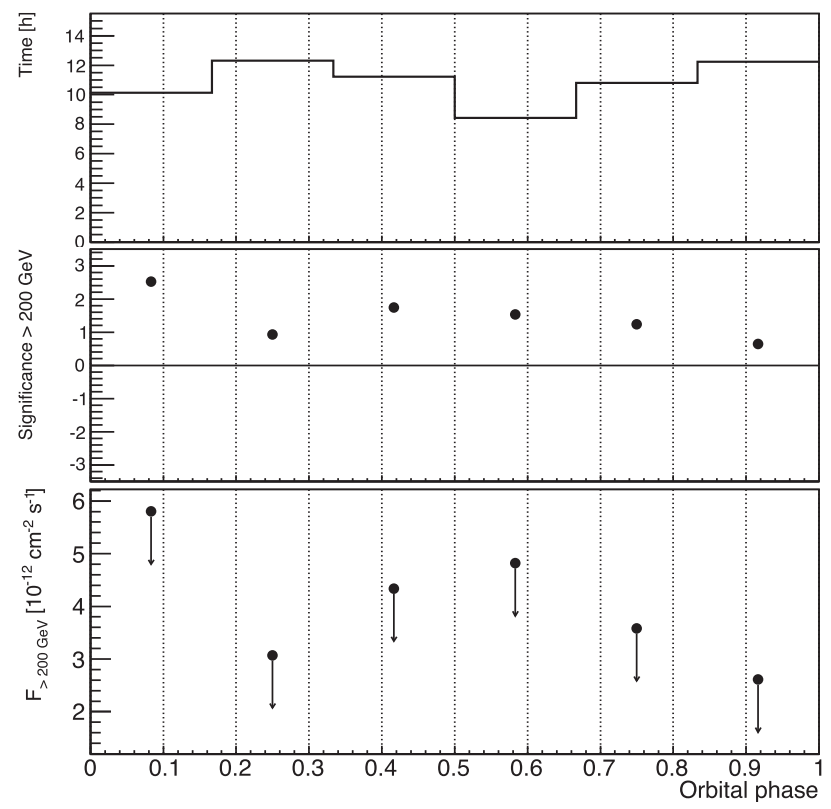

Figure 5. Analysis of B1957+20 in bins of orbital phase: total observation time spent at a given phase (top panel), the corresponding significance of excess (middle panel) and the 95 per cent confidence level upper limit on the integral flux (bottom panel) above $200 \mathrm{GeV}$.

$0-0.17$. After correcting for the number of trials corresponding to the binning in the orbital phase, the significance drops to $1.8 \sigma$, making it consistent with a random fluctuation. Values of the upper limits in individual bins of orbital phase range from $2.6 \times 10^{-12} \mathrm{~cm}^{-2} \mathrm{~s}^{-1}$ (1.1 per cent C.U.) up to $5.8 \times 10^{-12} \mathrm{~cm}^{-2} \mathrm{~s}^{-1}$ (2.6 per cent C.U.).

According to some of the models the emission from the black widow nebula might appear as slightly extended for MAGIC. Assuming a 10 arcmin radius of the source with a flat-top distribution, we have computed the significance of the excess within a broader $\theta^{2}$ cut and obtained a slightly higher value of $2.2 \sigma$. As more background events are integrated with a broader cut, the upper limit on the flux from the extended source is worse, $3.7 \times 10^{-12} \mathrm{~cm}^{-2} \mathrm{~s}^{-1}$ (i.e. 1.6 per cent C.U.) above $200 \mathrm{GeV}$. We have also calculated the upper limit of the flux above $200 \mathrm{GeV}$ of such an extended source shifted by $\sim 13 \operatorname{arcmin}$ (see equation 2 ) opposite to the di-

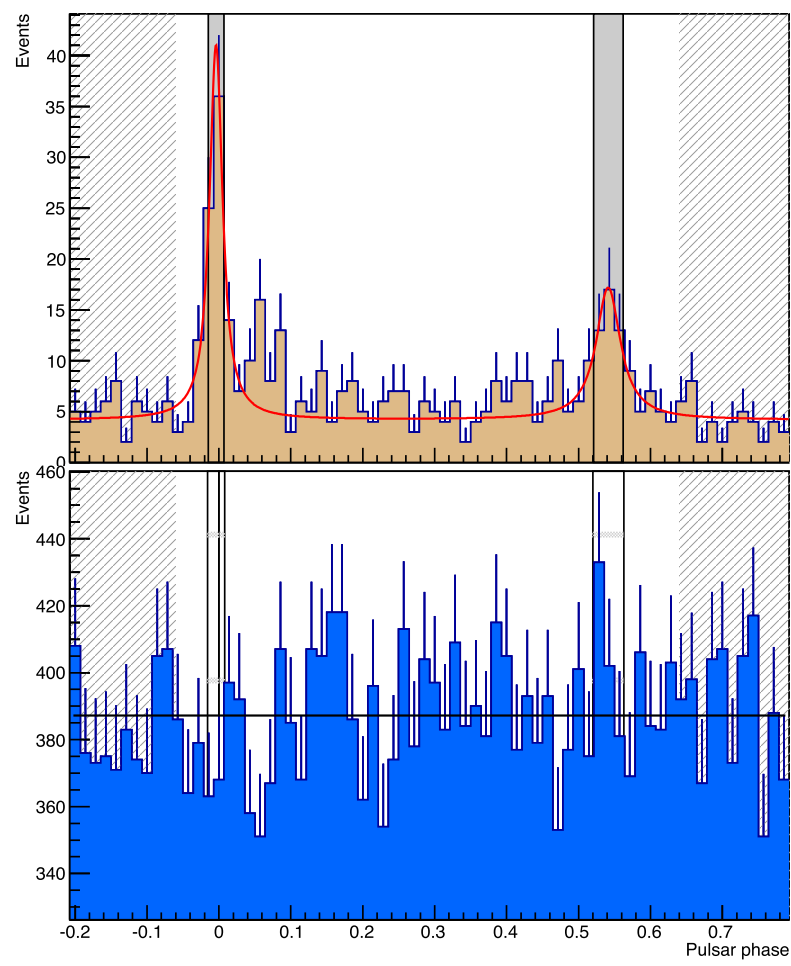

Figure 6. Light curve of the $\gamma$-ray emission from PSR B1957+20 using Fermi-LAT data above $1 \mathrm{GeV}$ (top panel) and MAGIC above $50 \mathrm{GeV}$ using data with zenith angles $<30^{\circ}$ (bottom panel). The phase 0 corresponds to the position of the main peak seen at radio wavelengths. The red line in the top panel represents the fitted Lorentzian functions. The shaded areas correspond to the phase intervals within the full widths at half-maximum of the fitted peaks, while the dashed regions represent the regions used to estimate the unpulsed background level. The horizontal black line in the bottom panel represents the fitted background level.

rection of the observed movement of the binary and obtained $1.8 \times$ $10^{-12} \mathrm{~cm}^{-2} \mathrm{~s}^{-1}$.

\subsection{Analysis of the pulsed emission}

To determine the characteristics of the B1957+20 light curve at energies as close as possible to the MAGIC energy range, we have phase folded the Fermi-LAT photons with energies above $1 \mathrm{GeV}$. The resulting light curve, after applying the energy-dependent angular cut described in Section 3, is shown in the top panel of Fig. 6. Above this energy, the two peaks are still clearly visible. We obtained a value of the bin-independent $H$-test parameter (de Jager \& Büsching 2010) of 179.5 corresponding to a pulsation significance of $11.8 \sigma$. Each peak was fitted with a Lorentzian symmetric function. The signal regions for the analysis of the MAGIC data were defined as the regions within the full widths at half-maximum (FWHM) of the fitted peaks. We considered background regions as the off-pulse, where no emission is expected from the pulsar, starting 2.5 FWHM away from the peak centres. Table 1 summarizes the signal and background regions later used for the analysis of MAGIC data. From now on, P1 and P2 will always be referred to as the values in Table 1 .

We fit the Fermi-LAT spectra above $100 \mathrm{MeV}$ for P1 and P2 using a power law with a cut-off function, defined as

$\frac{\mathrm{d} F}{\mathrm{~d} E}=N_{0}\left(\frac{E}{E_{0}}\right)^{-\alpha} \exp \left(-\left(E / E_{c}\right)^{b}\right)$, 
PSR B1957+20:P1

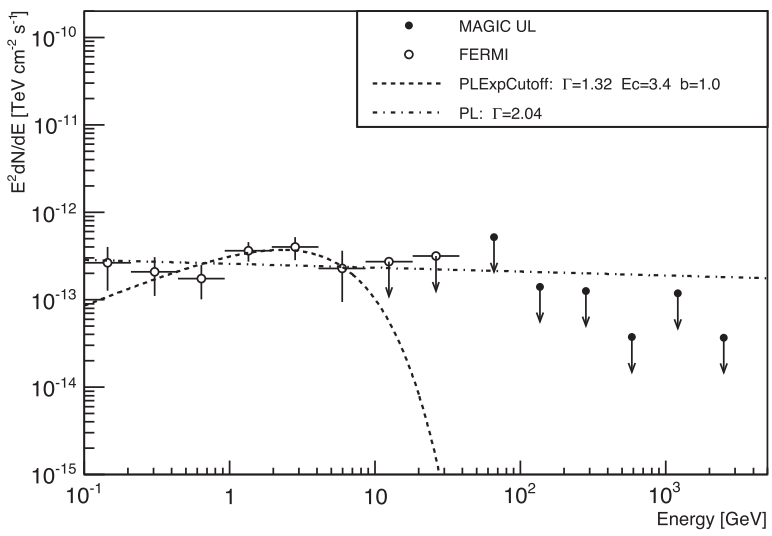

PSR B1957+20:P2

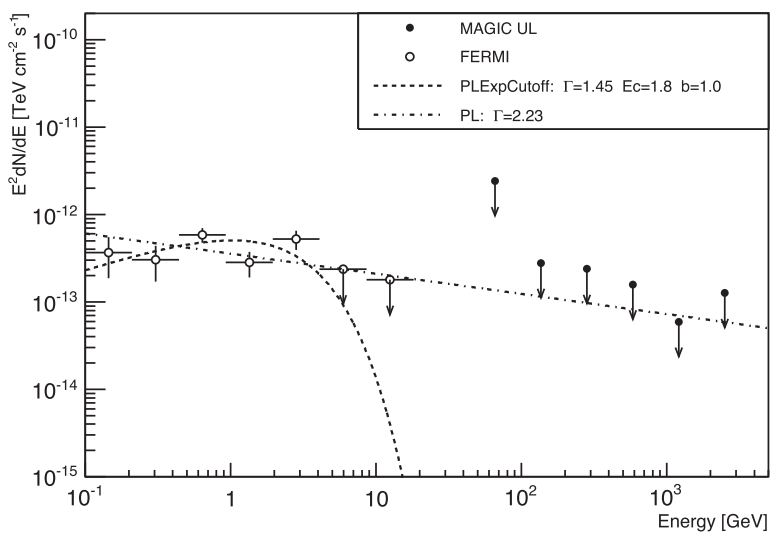

Figure 7. MAGIC upper limits for pulsed emission on the level of SED of B1957+20 and Fermi-LAT phase spectral energy points for P1 (top panel) and P2 (lower panel).

Table 2. Spectral parameters of the fits of the Fermi -LAT data above $100 \mathrm{MeV}$.

\begin{tabular}{lcccr}
\hline & $N_{0}$ & $\alpha$ & $E_{c}(\mathrm{GeV})$ & $b$ \\
\hline $\mathrm{P} 1$ & $4.1 \pm 0.6$ & $2.04 \pm 0.11$ & - & 0 \\
$\mathrm{P} 2$ & $5.9 \pm 0.7$ & $2.23 \pm 0.10$ & - & 0 \\
$\mathrm{P} 1$ & $5.6 \pm 1.3$ & $1.32 \pm 0.35$ & $3.4 \pm 1.8$ & 1 \\
$\mathrm{P} 2$ & $12.0 \pm 3.2$ & $1.45 \pm 0.31$ & $1.8 \pm 0.7$ & 1 \\
\hline Note: & the normalization & factor $N_{0}$ is given in units & of \\
$10^{-13} \mathrm{MeV}^{-1} \mathrm{~s}^{-1} \mathrm{~cm}^{-2}$. The quoted errors are statistical at a 1 $\sigma$ con- \\
fidence level. The systematic errors, as reported by the Fermi-LAT \\
team are of 14 per cent on $\alpha$ and 4 per cent on $E_{c}$ (Abdo et al. 2013).
\end{tabular}

where $E_{0}=0.8 \mathrm{GeV}$ is the energy scale, $\alpha$ the spectral index, $E_{c}$ the cut-off energy and $b$ the cut-off strength. In order to characterize the emission at high energies, we have considered two different cases: a simple power law $(b=0)$ and a power law with an exponential cut-off $(b=1)^{4}$. The results of the computed spectra in the phase regions defined in Table 1 are shown in Fig. 7 and tabulated in Table 2.

The MAGIC light curve for the pulsed emission above $50 \mathrm{GeV}$ is shown in the bottom panel of Fig. 6. No significant pulsation was found in the MAGIC data. The total number of excess events in the $\mathrm{P} 1$ and $\mathrm{P} 2$ phase regions are $23 \pm 47$, which corresponds to a

\footnotetext{
${ }^{4}$ We tried as well to fit the spectra leaving the $b$ parameter of equation (1) free, but the sharp cut-off did not allow us to constrain $b$.
}

Table 3. Significances of the search of pulsation in the MAGIC data for $\mathrm{P} 1, \mathrm{P} 2$ and for the sum of both peaks.

\begin{tabular}{lrrr}
\hline Energy range $(\mathrm{GeV})$ & $\mathrm{P} 1+\mathrm{P} 2$ & $\mathrm{P} 1$ & \multicolumn{1}{c}{$\mathrm{P} 2$} \\
\hline$\geq 50$ & $0.49 \sigma$ & $-0.91 \sigma$ & $1.28 \sigma$ \\
$50-100$ & $0.45 \sigma$ & $-1.36 \sigma$ & $1.54 \sigma$ \\
$100-200$ & $-0.06 \sigma$ & $0.09 \sigma$ & $-0.14 \sigma$ \\
\hline
\end{tabular}

significance of $0.5 \sigma$. The significances for $\mathrm{P} 1, \mathrm{P} 2$, and for the sum of both peaks are tabulated in Table 3 for different energy ranges.

The MAGIC differential upper limits computed for the pulsed emission are shown in Fig. 7 by black arrows. The spectral indices used for the upper limits computation were obtained from the powerlaw fits of the Fermi-LAT data shown in Table 2.

\section{COMPARISON WITH RADIATION MODELS}

$\mathrm{TeV} \gamma$-ray emission is already observed from several nebulae around classical pulsars which are formed in explosions of massive stars. Such nebulae are usually confined to a relatively small volume by the surrounding dense matter from the supernova remnant or the pre-supernova environment. Therefore, the interaction of relativistic electrons with the dense radiation field of soft photons takes place in a small volume around the pulsar. On the other hand, the only variable, rotation-powered pulsar observed in the $\mathrm{TeV} \gamma$ rays (PSR 1259-63; Aharonian et al. 2005) forms a binary system with a massive Be-type star. This star creates a strong target for electrons at the periastron passage. In the case of binary systems containing MSPs, the situation seems to be different. As MSPs are only found within low-mass binaries, the radiation fields created by their companion star are of lower density. Consequently, the production of $\gamma$-rays via the Comptonization process may be less efficient. In order to constrain the possible high-energy radiation processes around MSPs, we performed observations of the classical MSP binary system B1957+20. In fact, observation of non-thermal X-ray emission from this binary system indicates the presence of relativistic electrons within the binary system itself and in the extended region around it in the nebula. Therefore, some scenarios for the $\mathrm{TeV} \gamma$-ray emission from the MSP binary systems were suggested. Moreover, recent discovery of pulsed $\gamma$-ray emission observed up to $\sim 1 \mathrm{TeV}$ from the Crab pulsar (Aliu et al. 2008, 2011; Aleksić et al. 2012; Ansoldi et al. 2016) stimulates searches for such pulsed emission component also in the MSPs. The constraints provided by the observations reported in this paper on $\gamma$-ray emission from $\mathrm{B} 1957+20$ are discussed in this section.

\subsection{Gamma-rays from the bow shock nebula}

The non-thermal $\mathrm{X}$-ray radiation and $\mathrm{H} \alpha$ emission argue that the MSP binary system B1957+20 is surrounded by the bow shock nebula within which relativistic electrons are likely to be accelerated. In fact, the observed non-thermal X-ray emission extending up to $\sim 10 \mathrm{keV}$, if produced in the synchrotron process in the magnetic field of the nebula of the order of a few $\mu \mathrm{G}$ (expected within the MSP wind nebula), requires the existence of electrons with energies of the order of $\sim 100 \mathrm{TeV}$ (Cheng et al. 2006; Bednarek \& Sitarek 2013b). The problem appears whether such electrons are able to interact efficiently in the vicinity of the binary system, producing $\mathrm{TeV} \gamma$-ray fluxes observable by the present Cherenkov instruments. Bednarek \& Sitarek (2013b) consider the model for such bow shock nebula around the binary system B1957+20 in 
which electrons are assumed to be accelerated in the pulsar wind region. Electrons diffuse within the large scale nebula (of the order of parsecs) Comptonizing the cosmic microwave background and the infrared and optical diffuse radiation in the Galactic disc. The synchrotron and IC $\gamma$-ray spectra, calculated in terms of this model, depend on the pulsar and interstellar medium parameters. Some of these parameters can be constrained using observations of the nonthermal X-ray emission of this binary system (Huang et al. 2012).

We first compare the upper limits on the extended $\mathrm{TeV} \gamma$-ray emission from the binary system B1957+20 derived in Section 4, with the $\gamma$-ray spectra expected in terms of the model discussed by Bednarek \& Sitarek (2013b) under the assumption that the whole $\mathrm{X}$-ray emission is produced within this model. The Fermi-LAT measurement does not constrain the model, as the obtained flux upper limits overshoot model curve by an order of magnitude even for point-like emission. The model predicts the existence of an extended $\mathrm{TeV}$ source towards this binary system with the radius of the order of $\sim 10 \mathrm{pc}$. Assuming the distance to the binary system being $2.5 \mathrm{kpc}$ (consistent with the lower limit $\sim 2 \mathrm{kpc}$; van Kerkwijk et al. 2011), this corresponds to the angular extent of $\sim 13.8 \mathrm{arcmin}$. Moreover, the source is likely to be displaced from the present location of the binary system by the distance determined by the cooling time-scale of electrons on the IC scattering of the infrared radiation from the Galactic disc and the fast movement of the binary system through the interstellar space (see section 2 in Bednarek \& Sitarek 2013b).

The dimension of this $\gamma$-ray source is then estimated on $D \approx 8.5$ $\times 10^{7} / \gamma_{\mathrm{e}} \approx 42.5\left(E_{\mathrm{e}} / \mathrm{TeV}\right)^{-1} \mathrm{pc}$. It can be expressed by the energy of produced $\gamma$-rays applying the approximate relation between the energies of electrons and $\gamma$-rays produced by them, $E_{\gamma} / \mathrm{TeV}=\varepsilon \gamma_{\mathrm{e}}^{2} \approx$ $0.04\left(E_{\mathrm{e}} / \mathrm{TeV}\right)^{2}$, assuming an energy of the infrared photons equal to $\varepsilon \approx 0.01 \mathrm{eV}$. Then, the diameter of the source is estimated on

$D \approx 8.5 / \sqrt{E_{\gamma} / \mathrm{TeV}} \mathrm{pc}$.

Such a $\gamma$-ray source at the distance of $2.5 \mathrm{kpc}$ has an angular radius of $\sim 6$ arc min at energy of $1 \mathrm{TeV}$, and thus is well contained within the assumed 10 arc min radius of the extended source analysis.

The synchrotron X-ray spectrum from the tail in the nebula, extending up to $\sim 10 \mathrm{keV}$, puts constraints on the magnetization parameter of the pulsar wind, $\sigma \gtrsim 0.01$ (see section 3 in Bednarek \& Sitarek 2013b). In this model, the synchrotron emission and inverse Compton (IC) $\gamma$-ray emission from the extended nebula is produced by the same leptons. Therefore, the observed non-thermal $\mathrm{X}$-ray emission can be used as the normalization of the electron content within the nebula. We estimate that about 10 percent of the pulsar spin-down energy has to be converted to the relativistic leptons in the nebula. Still, the TeV emission predicted in this model lies below the MAGIC upper limits, derived for the extended source with a radius of $10 \mathrm{arcmin}$ (see Fig. 8). The predictions of the model are barely consistent with the obtained upper limits on the flux.

\subsection{Gamma-rays from the inner mixed wind nebula}

In general, nebulae around black widow MSP binary systems are expected to have quite complicated structure since, due to the Coriolis force, the pulsar wind can partially mix with the wind from the companion star in the equatorial region of the binary system (e.g. Bednarek \& Sitarek 2013a; Zabalza et al. 2013). Therefore, it is expected that in this region, the pulsar wind overtakes the slow stellar wind creating the collision region at some distance from the binary system. Then, the pulsar wind becomes loaded with the matter of the stellar wind. The mixed winds move together relatively slowly away from the binary system. Therefore, electrons, accelerated or

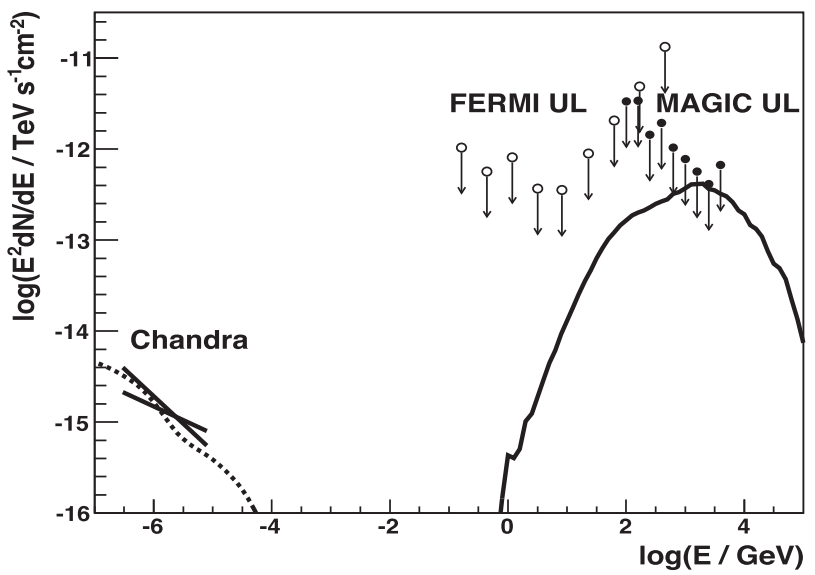

Figure 8. Comparison of the upper limits on the $\gamma$-ray emission from the nebula around the black widow binary pulsar B1957+20 obtained with MAGIC (10 arcmin radius extension assumed, filled points), Fermi-LAT (point-like, open points) and the X-ray tail emission detected by Chandra (Huang et al. 2012, thick bow-tie) with the predictions of the bow shock nebula model (region I) by Bednarek \& Sitarek (2013b) (solid and dotted lines). Assumed model parameters: leptons are injected from the pulsar wind region using a power-law spectrum with an index of 2.5 between 3 and $90 \mathrm{TeV}$, the magnetization parameter of the pulsar wind $\sigma=0.01$, the shock in the wind is located at a distance of $10^{16} \mathrm{~cm}$ from the pulsar and the minimum magnetic field strength within the nebula is $0.5 \mu \mathrm{G}$.

captured in such a collision region of the winds close to the radiation field of the companion star, can Comptonize efficiently the radiation from the hot part of the companion star. As a result, more efficient production of the high-energy $\gamma$-rays is expected than in the case of rectilinear propagation of leptons through the binary system (see table 2 in Bednarek \& Sitarek 2013a for parameters describing such scenario in the case of B1957+20). Since the collision region is outside of the binary system, this $\mathrm{TeV} \gamma$-ray emission should be steady, point-like (i.e at a distance of $\sim 2 \times 10^{12} \mathrm{~cm}$ or larger from the binary system) and centred on the present location of the binary system B1957+20.

Following the model of Bednarek \& Sitarek (2013a), we performed numerical calculations of expected $\mathrm{TeV} \gamma$-ray fluxes assuming that electrons are injected into the mixed wind region from the pulsar (or its surroundings) with a close to monoenergetic spectrum. Leptons with the $\mathrm{TeV}$ energies are predicted to escape from the inner pulsar magnetospheres (e.g. Sturrock 1971; Ruderman \& Sutherland 1975). The electron spectra, dominated energetically at the highest energies, are also expected to originate in the close vicinity of the pulsars. For example, the $\mathrm{GeV} \gamma$-ray synchrotron flares from the Crab nebula show very flat spectra that have to be produced by leptons with spectra dominated at the highest energies (Abdo et al. 2011; Tavani et al. 2011). The level of $\gamma$-ray emission in this model is determined by the product, $\Delta_{\text {mix }} \varepsilon$, of the solid angle covered by the mixed winds, $\Delta_{\text {mix }}$, and the energy conversion efficiency from the pulsar to relativistic electrons, $\varepsilon$. In the case of B1957+20, $\Delta_{\text {mix }}$ is estimated as $\sim 0.82$ (Bednarek \& Sitarek 2013a). We compare the upper limits derived for the case of the point-like $\mathrm{TeV} \gamma$-ray source towards B1957+20 with the results of calculations of the $\mathrm{TeV} \gamma$-ray fluxes for $\Delta_{\mathrm{mix}} \varepsilon=0.1$ (see Fig. 9) and other basic parameters of B1957+20 following the tables 1 and 2 in Bednarek \& Sitarek (2013a). Based on this comparison, we estimate the upper limit on the acceleration efficiency of electrons in terms of such a model in the range $\varepsilon \leq 2-10$ per cent for the mono-energetic electrons with energies in the range $0.3-10 \mathrm{TeV}$. Thus, the acceleration process of mono-energetic leptons to energies in the range 


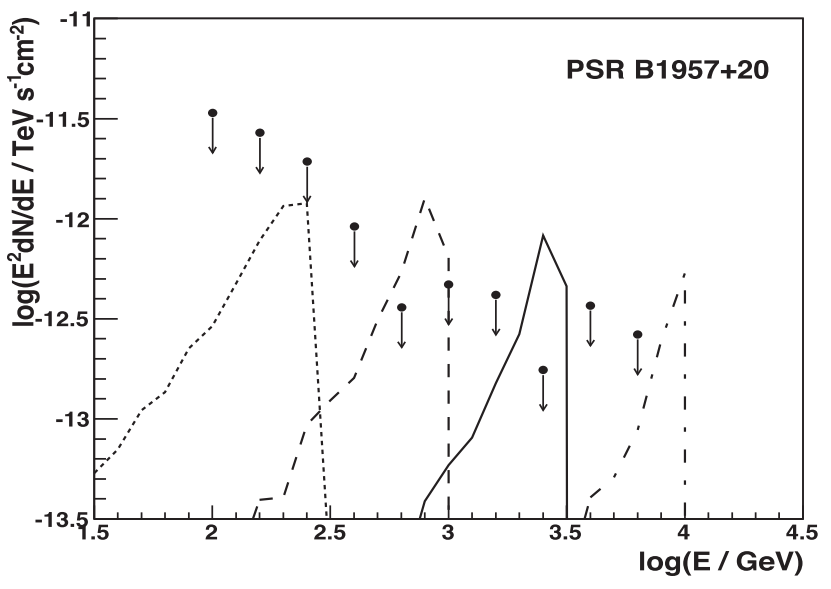

Figure 9. The comparison of the upper limits on the point-like $\mathrm{TeV} \gamma$-ray emission from the inner part of nebula around the black widow binary pulsar B1957+20 with the predictions of the mixed wind nebula model (region II). The leptons in the model are accelerated with the mono-energetic spectrum with energies equal to $300 \mathrm{GeV}$ (dotted curve), $1 \mathrm{TeV}$ (dashed), $3 \mathrm{TeV}$ (solid) and $10 \mathrm{TeV}$ (dot-dashed). It is assumed that the factor, describing the level of $\gamma$-ray emission, is equal to $\Delta_{\text {mix }} \varepsilon=0.1$ (see Section 4.2 for details).

covered by the MAGIC telescope energy range cannot be very efficient. We also have calculated the $\mathrm{TeV} \gamma$-ray fluxes for B1957+20 in the case of injection of electrons with the power-law spectrum. They are clearly on a lower level than the upper limits on the $\mathrm{TeV}$ $\gamma$-ray point-like source towards B1957+20 (in Fig. 4). Therefore, the point-like $\mathrm{TeV} \gamma$-ray emission from $\mathrm{B} 1957+20$, produced by electrons with the power-law spectrum in terms of this model, is predicted to be below the upper limits derived here based on the MAGIC telescopes observations.

\subsection{Modulated gamma-rays from the binary system}

The MSP binary systems of the black-widow-type are compact enough that the energy released by the pulsar can be responsible for the effective evaporation of the companion star and production of a relatively strong stellar wind (e.g. Ruderman, Shaham \& Tavani 1989). Moreover, the pulsar efficiently heats up a part of the stellar surface. The increased temperature in the part of the stellar surface results in a modulation of the optical emission from the companion star (e.g. Breton, van Kerkwijk \& Roberts 2013). The interaction of the pulsar and stellar winds creates a transition region (a shock structure) at a distance $\sim 5$ times larger than the size of the companion star $\left(10^{10} \mathrm{~cm}\right)$ in which particles can be accelerated (Harding \& Gaisser 1990; Arons \& Tavani 1993; Wadiasingh et al. 2015). It has been recently argued that the stellar and pulsar winds can mix efficiently in this transition region. Then, accelerated electrons might have enough time to Comptonize stellar radiation to the $\mathrm{TeV}$ energies and produce detectable $\gamma$-ray fluxes (Bednarek 2014). In such a case, it is expected that the radiation processes within MSP binary systems could occur similarly to those observed in the $\mathrm{TeV}$ $\gamma$-ray binaries, such as LS5039 or LSI $303+61$. The maximum energy of electrons accelerated in such a wind collision region is limited by their advection from the wind collision region and/or the synchrotron process. They depend on a few free parameters of the model from which two, namely the magnetization parameter of the pulsar wind, $\sigma$, and the velocity of the mixed pulsar/stellar wind, are not presently constrained by the observations. The $\sigma$ parameter cannot be too large in order not to dominate the electron energy losses by the synchrotron process. On the other hand, it can-

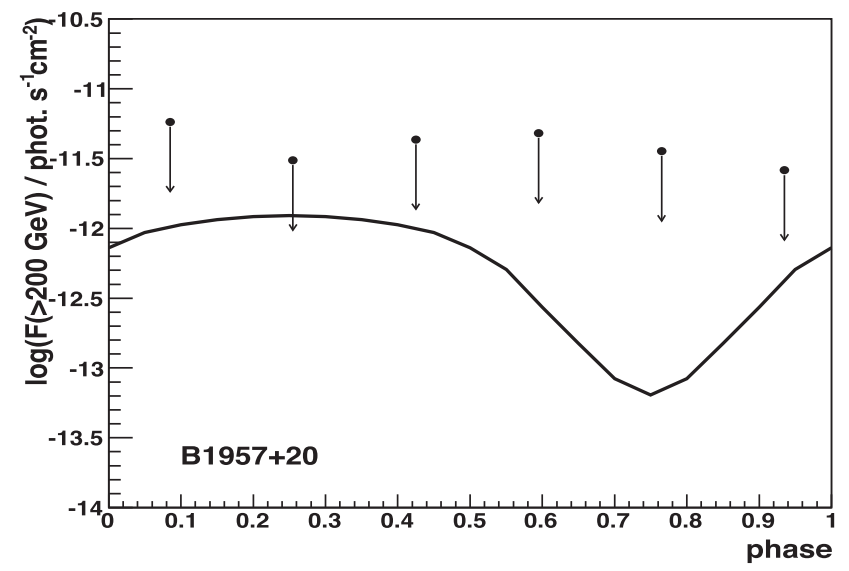

Figure 10. The comparison of the $\gamma$-ray light curve, folded with the orbital period of the system, at energies above $200 \mathrm{GeV}$, expected from the MSP binary system B1957+20 in terms of the IC model (region III) discussed by Bednarek (2014), with the upper limits on the $\gamma$-ray flux from this binary system at specific range of phases. The predicted $\gamma$-ray fluxes are obtained from the normalization of the synchrotron spectra, produced by the same population of electrons, to the observed modulated X-ray emission from this binary (see Huang et al. 2012).

not be also too low in order to allow acceleration of electrons to $\mathrm{TeV}$ energies.

In order to interpret the MAGIC measurement of B1957+20, we choose a magnetization parameter $\sigma=10^{-4}$, a mixed wind velocity $v_{\mathrm{w}}=10^{10} \mathrm{~cm} \mathrm{~s}^{-1}$, and other parameters of the model following (Bednarek 2014). The model assumes that electrons in the wind collision region are accelerated with a power-law spectrum with spectral index -2 and that 1 percent of the rotational energy loss rate is transferred to relativistic electrons. Note that the IC $\gamma$-ray emission expected in this model does not depend strongly on the values of $\sigma$ and $v_{\mathrm{w}}$ in contrast to the synchrotron emission (see fig. 2 ab in Bednarek 2014). The $\gamma$-ray emission produced by these electrons is expected to be modulated with the period of the binary system since the stellar radiation field is anisotropic with respect to the acceleration region of electrons. It was predicted to be on the sensitivity limit of the extensive observations with the current generation of the Cherenkov telescopes at the binary phase when the pulsar is behind the companion star (Bednarek 2014). We have performed calculations of the $\gamma$-ray emission in terms of this model for the MSP binary system B1957+20 and for the set of the parameters described above, also see table 1 in Bednarek (2014). In Fig. 10, we compare the predicted, orbital phase folded, $\gamma$-ray light curve with the upper limits obtained by MAGIC. The largest TeV $\gamma$-ray fluxes are predicted by the model for the orbital phase of 0.25 (i.e. when the pulsar is behind the star). The integral $\gamma$-ray flux above $200 \mathrm{GeV}$ expected in this model, equal to $1.4 \times 10^{-12} \mathrm{~cm}^{-2} \mathrm{~s}^{-1}$ is below the MAGIC upper limit of $3.1 \times 10^{-12} \mathrm{~cm}^{-2} \mathrm{~s}^{-1}$. Therefore, the present observations with the MAGIC telescopes allow us to conclude that less than 3 per cent of the rotational energy loss rate of the pulsar within the binary system B1957+20 has been transferred to relativistic electrons accelerated at the region of collision of the pulsar and stellar winds as postulated in the abovementioned model.

\subsection{Pulsed gamma-rays from the pulsar}

Recent discovery of pulsed $\sim 100 \mathrm{GeV} \gamma$-ray emission from the Crab pulsar (Aliu et al. 2011; Aleksić et al. 2012; Ansoldi et al. 2016) provided new constraints on the models for the GeV-TeV $\gamma$-ray emission from the pulsar magnetospheres. Different scenarios 
and radiation mechanisms have been proposed as responsible for this unexpected emission. For example, Lyutikov, Otte \& McCann (2012) and Aleksić et al. (2012) argue that this pulsed emission component is produced in the Comptonization process occurring within the outer gap scenario. It is also suggested that electrons, accelerated close to the light cylinder radius (at a distance of $7 \times$ $10^{6} \mathrm{~cm}$ from the black widow pulsar), can produce $\gamma$-rays in the curvature process in the distorted magnetic field (Bednarek 2012; Bogovalov 2014) or due to IC scattering of thermal radiation from the NS surface (Osmanov \& Rieger 2017). This emission could also come from the outside of the light cylinder radius, from the region of the equatorial current sheet (e.g. Arka \& Dubus 2013; Chen \& Beloborodov 2014; Uzdensky \& Spitkovsky 2014) or at the distance of a few tens of the light cylinder radii as a result of Comptonization of the X-rays produced in the inner magnetosphere (Aharonian, Bogovalov \& Khangulyan 2012; Petri 2012). Moreover, Bednarek (2012) suggests that the middle-aged pulsars of the Vela type and some extreme MSPs should also be able to produce tails of pulsed high-energy $\gamma$-ray emission extending to $\sim 50 \mathrm{GeV}$. In fact, such emission has been recently discovered from the Vela pulsar (Gajdus et al. 2015). However, such tails are not expected in the case of the older pulsars (e.g. Geminga type).

In Fig. 7, we compare the TeV upper limits obtained by MAGIC on the pulsed $\gamma$-ray emission from B1957+20 with the SED measured by Fermi-LAT. The MAGIC upper limits are well above the extrapolation of the fitted exponential cut-offs for any of the two emission peaks.

\section{CONCLUSIONS}

No signal at the $\mathrm{TeV}$ energies has been detected from the black widow binary system B $1957+20$ and its surrounding by the MAGIC telescopes. The upper limits above $200 \mathrm{GeV}$ obtained from these data are $\sim 1.3$ per cent C.U. for a point-like source and $\sim 1.6$ per cent C.U. assuming its extension of $10 \mathrm{arcmin}$. They are not very restrictive due to a small positive excess in the direction of B1957+20. The orbital phase analysis also does not show any clear hint of emission localized at any particular orbital phase. The pulsar phase analysis also does not show any positive excess above $50 \mathrm{GeV}$. The derived upper limits for pulsed emission do not allow us to constrain a very high energy extension of the Fermi-LAT spectra. We have compared these upper limits with the predictions of a few models that postulate production of the $\mathrm{TeV} \gamma$-rays within different parts of the binary system B1957+20 or its vicinity. From the comparison of the model for the extended bow shock nebula around the black widow binary system (see Section 5.1), we confirm that the magnetization parameter of the pulsar wind in such an extended nebula formed by the freely expanding wind should be larger than $\sigma \sim$ 0.01 . Thus, the magnetization of the winds around MSPs seems to be clearly larger than the magnetization of the winds produced by young classical pulsars of the Crab type, equal to $\sigma \sim 0.003$ (Kennel \& Coroniti 1984), but more similar to the magnetization of the Vela-type pulsars in the range $0.05<\sigma<0.5$ (Sefako \& de Jager 2003). On the other hand, the transfer of energy from the magnetized wind to relativistic leptons might occur more efficiently in the equatorial region of the binary system where the pulsar and stellar winds can mix efficiently (see Section 5.2). The MAGIC upper limit, derived on a point-like source in the direction of B1957+20, allows us to constrain the efficiency of energy conversion from the pulsar to relativistic electrons in this scenario to be below $\sim 2-10$ per cent, provided that they are mono-energetic with energies in the range $0.3-10 \mathrm{TeV}$. The $\mathrm{TeV} \gamma$-rays are expected to be also efficiently produced in the region of collisions of the pulsar and companion star winds within the binary system (see Section 5.3). The largest $\gamma$-ray fluxes are predicted in this scenario when the pulsar is behind the companion star. However, the MAGIC 95 per cent confidence level upper limits are a factor of $\sim 2$ above predictions of such a wind collision model in the case of efficient conversion of the pulsar wind energy into relativistic leptons in the collision region and likely parameters of the companion star wind. Therefore, more sensitive observations of this binary system with the future CTA (further supported by a weak hint of excess seen by MAGIC at the level of $\sim 2 \sigma$ ) will be needed in order to detect this modulated $\gamma$-ray emission from B1957+20. In the case of $\gamma$-ray emission from pulsar itself, MAGIC observations rule out a simple powerlaw extrapolation of the $\mathrm{P} 1$ pulsed emission through the sub-TeV energy range (Section 5.4). We conclude that MAGIC upper limits on the $\gamma$-ray emission from the MSP binary B1957+20 are generally consistent with the predictions of the present models. Most of these models predict $\gamma$-ray fluxes that depend on the strength of the radiation field created by the companion star. Therefore, future, more sensitive observations of the MSP binary systems of the redback type (e.g. such as PSR J1816+4510) are expected to provide stronger constraints on the acceleration processes of leptons within the binaries and their surroundings.

\section{ACKNOWLEDGEMENTS}

We would like to thank the Instituto de Astrofísica de Canarias for the excellent working conditions at the Observatorio del Roque de los Muchachos in La Palma. The financial support of the German BMBF and MPG, the Italian INFN and INAF, the Swiss National Fund SNF, the ERDF under the Spanish MINECO (FPA201569818-P, FPA2012-36668, FPA2015-68378-P, FPA2015-69210C6-2-R, FPA2015-69210-C6-4-R, FPA2015-69210-C6-6-R, AYA 2015-71042-P, AYA2016-76012-C3-1-P, ESP2015-71662-C2-2-P, CSD2009-00064) and the Japanese JSPS and MEXT is gratefully acknowledged. This work was also supported by the Spanish Centro de Excelencia 'Severo Ochoa' SEV-2012-0234 and SEV-20150548, and Unidad de Excelencia 'María de Maeztu' MDM-20140369, by the Croatian Science Foundation (HrZZ) Project 09/176 and the University of Rijeka Project 13.12 .1 .3 .02 , by the DFG Collaborative Research Centers SFB823/C4 and SFB876/C3, and by the Polish Narodowe Centrum Nauki grant 2016/22/M/ST9/00382. This work is partially supported by the grant through the Polish Narodowe Centrum Nauki No. 2014/15/B/ST9/04043. The Nançay Radio Observatory is operated by the Paris Observatory, associated with the French Centre National de la Recherche Scientifique (CNRS).

\section{REFERENCES}

Abdo A. A. et al., 2010, ApJ, 708, 1254

Abdo A. A. et al., 2011, Science, 331, 739

Abdo A. A. et al., 2013, ApJS, 208, 17

Acero F. et al., 2015, ApJS, 218, 23

Aharonian F. A. et al., 2005, A\&A, 442, 1

Aharonian F. A., Bogovalov S. V., Khangulyan D., 2012, Nature, 482, 507

Aleksić J. et al., 2012, A\&A, 540, 69

Aleksić J. et al., 2016a, Astropart. Phys., 72, 61

Aleksić J. et al., 2016b, Astropart. Phys., 72, 76

Aliu E. et al., 2008, Science, 322, 1221

Aliu E. et al., 2011, Science, 334, 69

Ansoldi S. et al., 2016, A\&A, 585, A133 
Arka I., Dubus G., 2013, A\&A, 550, A101

Arons J., Tavani M., 1993, ApJ, 403, 249

Arzoumanian Z., Fruchter A. S., Taylor J. H., 1994, ApJ, 426, L85

Bednarek W., 2012, MNRAS, 424, 2079

Bednarek W., 2014, A\&A, 561, A116

Bednarek W., Sitarek J., 2013b, A\&A, 550, A39

Bednarek W., Sitarek J., 2013a, MNRAS, 430, 2951

Bogovalov S. V., 2014, MNRAS, 443, 2197

Breton R. P., van Kerkwijk M. H., Roberts M. S. E., 2013, ApJ, 769, 108

Brink C. et al., 1990, ApJ, 364, L37

Buccheri R. et al., 1996, A\&AS, 115, 305

Chen A. Y., Beloborodov A. M., 2014, ApJ, 794, 24

Cheng K. S., Taam R. E., Wang W., 2006, ApJ, 641, 427

de Jager O. C., Büsching I, 2010, A\&A, 517, L9

Fruchter A. S., Stinebring D. R., Taylor J. H., 1988, Nature, 333, 237

Fruchter A. S. et al., 1996, ApJ, 443, 21

Fruck C., Gaug M., 2015, Eur. Phys. J. Web Conf., 89, 02003

Gajdus M., Djannati Ataï A., Chrétien M., Giavitto G., Holler M., Rudak B., Tavernier T., Venter C., 2015, Proc. Sci., Pulsations from the Vela pulsar down to $20 \mathrm{GeV}$ with H.E.S.S. II, SISSA, Trieste, PoS(ICRC2015) 841

Guillemot L. et al., 2012, ApJ, 744, 33

Harding A. K., Gaisser T. K., 1990, ApJ, 358, 561

Hobbs G. B., Edwards R. T., Manchester R. N., 2006, MNRAS, 369, 655

Huang R. H. H., Becker W., 2007, A\&A, 463, L5

Huang R. H. H., Kong A. K. H., Takata J., Hui C. Y., Lin L. C. C., Cheng K. S., 2012, ApJ, 760, 92

Kennel C. F., Coroniti F. V., 1984, ApJ, 283, 710

Kulkarni S. R., Hester J. J., 1988, Nature, 335, 801

Li T.-P., Ma Y.-Q., 1983, ApJ, 272, 317

Lyutikov M., Otte N., McCann A., 2012, ApJ, 754, 33

Osmanov Z., Rieger F. M., 2017, MNRAS, 464, 1347

Petri J., 2012, MNRAS, 424, 2023

Raubenheimer B. C., Brink C., de Jager O. C., Nel H. I., 1995, ApJ, 444, 419

Reynolds M. T., Callanan P. J., Fruchter A. S., Torres M. A. P., Beer M. E., Gibbons R. A., 2007, MNRAS, 379, 1117

Rolke W. A., López A. M., Conrad J., 2005, Nucl. Instrum. Methods Phys. Res. A, 551, 493

Ruderman M. A., Sutherland P. G., 1975, ApJ, 196, 51

Ruderman M., Shaham J., Tavani M., 1989, ApJ, 336, 507

Sefako R. R., de Jager O. C., 2003, ApJ, 593, 1013

Stappers B. W., Gaensler B. M., Kaspi V. M., van der Klis M., Lewin W. H. G., 2003, Science, 299, 1372

Sturrock P. A., 1971, ApJ, 164, 529

Tavani M. et al., 2011, Science, 331, 736

Uzdensky D. A., Spitkovsky A., 2014, ApJ, 780, 3

van Kerkwijk M. H., Breton R. P., Kulkarni S. R., 2011, ApJ, 728, 95

van Paradijs J. et al., 1988, Nature, 334, 684

Wadiasingh Z., Harding A. K., Venter C., Böttcher M., 2015, Proc. Sci., Orbitally Modulated Emission at Intrabinary Shocks in Millisecond Pulsar Binaries. SISSA, Trieste, PoS(HEASA2015)029

Wu E. M. H. et al., 2012, ApJ, 761, 181

Zabalza V., Bosch-Ramon V., Aharonian F., Khangulyan D., 2013, A\&A, 551, A17

Zanin R. et al., 2013, Proc. 33rd ICRC, Rio de Janeiro, Brazil, 773
${ }^{1}$ ETH Zurich, CH-8093 Zurich, Switzerland

${ }^{2}$ Università di Udine, and INFN Trieste, I-33100 Udine, Italy

${ }^{3}$ Japanese MAGIC Consortium, ICRR, The University of Tokyo, Department of Physics and Hakubi Center, Kyoto University, Tokai University, The University of Tokushima, Japan

${ }^{4}$ INAF National Institute for Astrophysics, I-00136 Rome, Italy

${ }^{5}$ Università di Padova and INFN, I-35131 Padova, Italy

${ }^{6}$ Croatian MAGIC Consortium, Rudjer Boskovic Institute, University of Rijeka, University of Split - FESB, University of Zagreb - FER, University of Osijek, Croatia

${ }^{7}$ Saha Institute of Nuclear Physics, 1/AF Bidhannagar, Salt Lake, Sector-1, Kolkata 700064, India

${ }^{8}$ Max-Planck-Institut für Physik, D-80805 München, Germany

${ }^{9}$ Centro Brasileiro de Pesquisas Físicas (CBPF/MCTI), R. Dr Xavier Sigaud, 150 - Urca, Rio de Janeiro RJ 22290-180, Brazil

${ }^{10}$ Universidad Complutense, E-28040 Madrid, Spain

${ }^{11}$ Inst. de Astrofísica de Canarias, E-38200 La Laguna, Tenerife, Spain

${ }^{12}$ Universidad de La Laguna, Dpto. Astrofísica, E-38206 La Laguna, Tenerife, Spain

${ }^{13}$ NASA Goddard Space Flight Center, Greenbelt, MD 20771, USA

${ }^{14}$ Department of Physics and Department of Astronomy, University of Maryland, College Park, MD 20742, USA

${ }^{15}$ Department of Astrophysics, University of Lodz, ul. Pomorska 149/153, 90-236 Lodz, Poland

${ }^{16}$ Deutsches Elektronen-Synchrotron (DESY), D-15738 Zeuthen, Germany

${ }^{17}$ Humboldt University of Berlin, Institut für Physik Newtonstr. 15, D-12489 Berlin, Germany

${ }^{18}$ University of Trieste and INFN Trieste, I-34127 Trieste, Italy

${ }^{19}$ Institut de Fisica d'Altes Energies (IFAE), The Barcelona Institute of Science and Technology, Campus UAB, E-08193 Bellaterra (Barcelona), Spain

${ }^{20}$ Università di Siena, and INFN Pisa, I-53100 Siena, Italy

${ }^{21}$ Universität Würzburg, D-97074 Würzburg, Germany

${ }^{22}$ Institute for Space Sciences (CSIC/IEEC), E-08193 Barcelona, Spain

${ }^{23}$ Technische Universität Dortmund, D-44221 Dortmund, Germany

${ }^{24}$ Finnish MAGIC Consortium: Tuorla Observatory, University of Turku and Astronomy Division, University of Oulu, Finnish Centre for Astronomy with ESO (FINCA), Finland

${ }^{25}$ Unitat de Física de les Radiacions, Departament de Física, and CERESIEEC, Universitat Autònoma de Barcelona, E-08193 Bellaterra, Spain

${ }^{26}$ Universitat de Barcelona, ICC, IEEC-UB, E-08028 Barcelona, Spain

${ }^{27}$ Japanese MAGIC Consortium

${ }^{28}$ Inst. for Nucl. Research and Nucl. Energy, BG-1784 Sofia, Bulgaria

${ }^{29}$ Università di Pisa, and INFN Pisa, I-56126 Pisa, Italy

${ }^{30}$ ICREA and Institute for Space Sciences (CSIC/IEEC), E-08193 Barcelona, Spain

${ }^{31}$ Laboratoire de Physique et Chimie de l'Environnement et de l'Espace, LPC2E, CNRS-Université d'Orléans, F-45071 Orléans, France

${ }^{32}$ Station de Radioastronomie de Nançay, Observatoire de Paris, CNRS/INSU, F-18330 Nançay, France

This paper has been typeset from a $\mathrm{T}_{\mathrm{E}} \mathrm{X} / \mathrm{LT} \mathrm{E} \mathrm{X}$ file prepared by the author. 